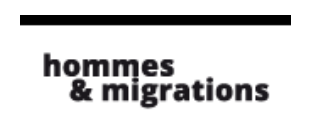

Hommes \& migrations

Revue française de référence sur les dynamiques

migratoires

1286-1287 | 2010

Les migrations subsahariennes

\title{
Reconstitution territoriale et codéveloppement
}

L'exemple de la diaspora sénégalaise

\section{Abdoul Hameth Ba}

\section{(2) OpenEdition}

1 Journals

\section{Édition électronique}

URL : http://journals.openedition.org/hommesmigrations/1750

DOI : 10.4000/hommesmigrations. 1750

ISSN : 2262-3353

Éditeur

Musée national de l'histoire de l'immigration

Édition imprimée

Date de publication : 1 juillet 2010

Pagination : 200-211

ISSN : 1142-852X

Référence électronique

Abdoul Hameth $\mathrm{Ba}$, «Reconstitution territoriale et codéveloppement », Hommes \& migrations [En ligne], 1286-1287 | 2010, mis en ligne le 01 juillet 2012, consulté le 30 avril 2019. URL : http:// journals.openedition.org/hommesmigrations/1750 ; DOI : 10.4000/hommesmigrations.1750 


\section{Reconstitution territoriale et codéveloppement L'exemple de la diaspora sénégalaise}

Par Abdoul Hameth Ba, maître de conférences en géographie, université d'Evry-Val d'Essonne ; membre du LHEST (Laboratoire histoire économique sociale et des techniques)

Éloignés de leur région d'origine, les migrants sahéliens participent aux processus de transformation territoriale et sociale aussi bien dans les zones d'émigration que dans les pays d'immigration. La concrétisation

de leurs projets liés à l'émigration témoigne de leur capacité à agir sur un espace à distance. Elle engage la fabrication progressive

de nouveaux territoires et de nouveaux modes de concertation. Cependant, dans le cadre institutionnel du codéveloppement, l'expérience des migrants n'est pas toujours valorisée. 
Avec l'évolution rapide du profil des migrants sahéliens et de leurs projets, des interrogations plus spécifiques se posent. Comment mesurer les interactions sociales et spatiales liées aux projets réalisés par ces acteurs délocalisés? Peut-on dire que ces migrants contribuent à l'émergence de territoires d'un type nouveau dans leur pays d'origine et dans les pays d'accueil? Peut-on considérer qu'ils introduisent directement ou indirectement une gouvernance participative d'un type nouveau, reflet d'une mise en réseau complexe tissée par-delà les frontières ?

Pour esquisser une réponse à ces questionnements, nous avons choisi d'analyser les processus migratoires dans l'espace sahélien, confronté ces trois dernières décennies à des tensions politiques et sociales et à des problèmes environnementaux très aigus. En effet, une forte croissance démographique (taux de natalité parfois supérieur à 40 pour 1000 ) conjuguée à une diminution des ressources naturelles (terres cultivables, zones de pêche, zones de pâturage, forêt ...) consécutive aux sécheresses de 1973-1974 et de 1983-1984 rendent la population sahélienne rurale très vulnérable. Les politiques d'aménagement et de développement rural préconisées par les gouvernements des États sahéliens ainsi que les aides internationales demeurent insuffisantes. Aussi, dans ces régions, l'émigration est-elle encore considérée comme l'une des solutions permettant à une partie de la population restée de survivre et de préserver les terroirs villageois.

Les stratégies des migrants en direction des zones de départ évoluent. Si au début des années soixante les migrants agissaient seuls, depuis quelques années ce n'est plus le cas. Ils collaborent avec des organisations non gouvernementales locales ou étrangères et/ou des collectivités territoriales européennes qui agissent dans le cadre de la coopération décentralisée. La mise en réseau de ces différents acteurs aux compétences variées et aux stratégies parfois divergentes signe l'avènement d'un mode nouveau de gestion des ressources naturelles et humaines. En effet, la définition, le choix, le financement et la gestion d'un projet nécessitent l'instauration de règles nouvelles de dialogue, de concertation, de prise de décision. Le partage des rôles entre sédentaires (gardiens des territoires anciens) et émigrés partis à la recherche du numéraire pour préserver ce qu'il reste du patrimoine naturel et culture est-il une spécificité des personnes originaires de l'Afrique rurale sahélienne ${ }^{(1)}$ ?

\section{Assurer sa place dans les pays d'immigration}

Les mouvements de population participent au brassage des peuples et donc des cultures dans ses différents aspects (langue, musique, art culinaire, religion, art vestimentaire, architecture). Dans les pays d'immigration, l'installation très longue des immigrés 
instaure une mixité sociale et ethnique, voulue ou subie, d'un type nouveau. En effet, en décidant de se fixer définitivement (au moins durant la vie active) dans un pays, les immigrés tentent de se "refaire un territoire". Autrement dit, de s'approprier un espace à l'intérieur duquel ils peuvent vivre ou (re)vivre une partie de leur culture (pratique des cultes). Ce processus de fabrication de territoires nouveaux est long et suscite des tensions entre la population autochtone et les immigrés.

La notion de "territoire" recouvre plusieurs dimensions : physique, juridique, économique, sociale, culturelle. Tout territoire résulte d'un processus d'appropriation d'une portion de l'étendue terrestre par un groupe social. Il est par conséquent marqué par des conflits plus ou moins longs, plus ou moins violents, plus ou moins visibles, avec d'autres groupes revendiquant éventuellement la même portion. En partant de l'idée que pour se maintenir sur son territoire et assurer la satisfaction de ses besoins tout groupe social doit décider d'un certain nombre d'aménagements, d'activités, dont la nature est liée à son niveau d'organisation et aux lois en vigueur, on peut comprendre le besoin des immigrés d'origine sahélienne ayant décidé de s'installer durablement en Europe de constituer progressivement leurs territoires.

\section{De nouvelles organisations de l'espace}

Les territoires des immigrés étaient des enclaves réduites et peu perceptibles dans les premières années d'installation (1960). Dans l'histoire de la migration ouestafricaine en France, les premiers arrivants sont installés dans des foyers isolés conçus essentiellement pour des travailleurs immigrés. Progressivement, les locataires s'organisent: de petites salles de prière sont aménagées dans les caves puis à l'intérieur des foyers, d'autres espaces de convivialité sont aussi aménagés : coins cuisine, restaurants tenus par des femmes, petits commerces, ateliers de tailleurs. On assiste alors à une diversification des activités. Par le regroupement familial qui s'accélère à la fin des années soixante-dix, une nouvelle configuration de la population immigrée se dessine. Épouse et enfants rejoignent le mari. C'est ainsi qu'apparaissent de nouveaux besoins, de nouvelles exigences. Les immigrés mariés sortent des foyers et s'installent dans des logements sociaux : on assiste alors à des processus d'agrégation voulue ou imposée.

Les immigrés savent que leur existence, leur épanouissement sont liés à leur possibilité de faire territoire, un territoire aux limites floues et molles, un territoire éphémère du fait des politiques de réhabilitation ou de rénovation urbaine, mais un territoire tout de même. La question en suspens reste la suivante : comment les 
Teritoire de PAlaef

Association intervillageoise transnationale de villages soninké 
nouvelles générations issues de l'immigration entendent préserver les territoires constitués par les primo-migrants? Allons-nous assister à une territorialisation des descendants d'immigrés? La constitution de territoires dans/par l'immigration peut conduire à une forme nouvelle d'exclusion de l'immigré et de ses descendants résultant d'un repli identitaire. En revanche, lorsque la proximité physique entre immigrés et population autochtone se passe bien, on assiste à une mixité culturelle. En définitive, tout processus de reconstitution de territoires dans l'immigration est lié à des paramètres variés (social, politique, économique, juridique, géographique). Cette forme d'ancrage territorial des immigrés signe d'une certaine manière une forme de citoyenneté recherchée, une volonté de participer à l'écriture de l'histoire du pays d'installation. De même, leur perception du territoire d'origine évolue à la lumière des méthodes de lectures spatiales acquises lors de leur trajet migratoire et dans le pays d'immigration. Par ailleurs, leurs projets en direction du territoire d'origine évoluent rapidement ces dernières années grâce, d'une part, à la dynamique du tissu associatif, dont les acteurs (hommes et femmes) sont de plus en plus expérimentés et instruits et, d'autre part, grâce aux politiques de solidarité gouvernementale ou non gouvernementale.

\section{Une émigration solidaire}

Les études sur les mobilités géographiques montrent que les migrants ont une propension à investir dans les pays d'origine ${ }^{(2)}$. La manifestation de l'attachement à la région d'origine et les types de projets des migrants varient en fonction de l'âge, du genre et du niveau d'instruction des migrants. Dans le cas de l'émigration sahélienne, et plus particulièrement de celle des ressortissants du bassin du fleuve Sénégal, on assiste ces quatre dernières décennies à des transformations sociales et territoriales dans les zones rurales.

En effet, ces changements sont dus aux réalisations d'équipements individuels (maison, petite entreprise...) ou collectifs (mosquée, puits, forage, barrage, matériel agricole, dispensaire, école, marché couvert...) et l'émergence d'un mouvement associatif très structuré en lien avec l'émigration. Si les premiers projets sont pensés totalement et financés intégralement par les migrants originaires d'un même village, plus récemment, des équipements collectifs sont réalisés grâce à un partenariat formel entre différents acteurs (organisations non gouvernementales, collectivités locales françaises, migrants...). Nous le constatons aujourd'hui, cette forme de coopération suscite de plus en plus d'engouement. Dans le cas du Sénégal par exemple, nous notons plus d'une centaine de partenariats avec les collectivités locales françaises. 
Cette forte solidarité des migrants à l'égard des territoires d'origine, tant en termes de réalisation d'équipements collectifs que de choix de leaders politiques locaux, est perceptible au Sénégal. D’ailleurs ces dix dernières années, les candidats aux élections locales ou nationales l'ont compris, ils se rendent en France pour obtenir le soutien des immigrés et, par conséquent, celui de leur famille restée au village. Le nombre et la nature des projets ont progressé grâce à l'augmentation du nombre des immigrés originaires du même village ou de la même circonscription administrative.

Dans le même temps, nous observons une diversification des types d'associations issues de l'immigration. Désormais, une collaboration formelle s'instaure entre migrants et structures d'animation, de formation et de développement. Nous observons aussi que, par effet de mimétisme, les associations se multiplient, les projets en direction du pays d'origine se diversifient, le tout impliquant le changement des échelles d'intervention. Nous notons aussi la création des associations intervillagoises qui s'étendent parfois sur plusieurs communautés rurales ou sur un espace transfrontalier. Même si ce type d'association est numériquement moins important, il témoigne de la part des migrants et des villageois d'une nouvelle perception de l'espace. 


\section{Quand des échanges créent un territoire}

C'est le cas de l'Alaef(Association de lutte anti-érosive sur la Falémé). Cette structure animée par d'anciens migrants est constituée de villages maliens et sénégalais qui partagent l'usage d'une langue commune, le soninké, et l'exploitation d'un cours d'eau, la Falémé, affluent du fleuve Sénégal. Grâce à l'appui technique du GRDR (Groupe de recherche et de développement rural) et d'un soutien financier des migrants, l'Alaef poursuit sa réflexion sur la dynamique économique de plusieurs villages en articulant les actions (protection de l'environnement, formation des jeunes, création d'emploi, désenclavement des villages...). L'espace économique de ces villages est géré par un réseau social structuré autour de l'Alaef dont le champ d'intervention est bien délimité. Cette zone d'intervention est un territoire en constitution comprenant un groupe social bien identifié et avec des objectifs précis. Ici, les stratégies des anciens migrants et des sédentaires ont permis l'émergence d'un territoire issu de la solidarité ethnique, par-delà les frontières (voir carte).

Une autre réalisation témoigne de la forte collaboration entre villageois et migrants pour gérer une ressource naturelle et revitaliser les activités artisanales. Il s'agit de la construction de mini barrages sur la Kolimbiné au Mali par l'Ordik (Organisation rurale pour le développement intégré de la Kolimbiné) dont le fondateur est un ancien immigré malien. Ces deux structures, et d'autres en Mauritanie, sont initiées

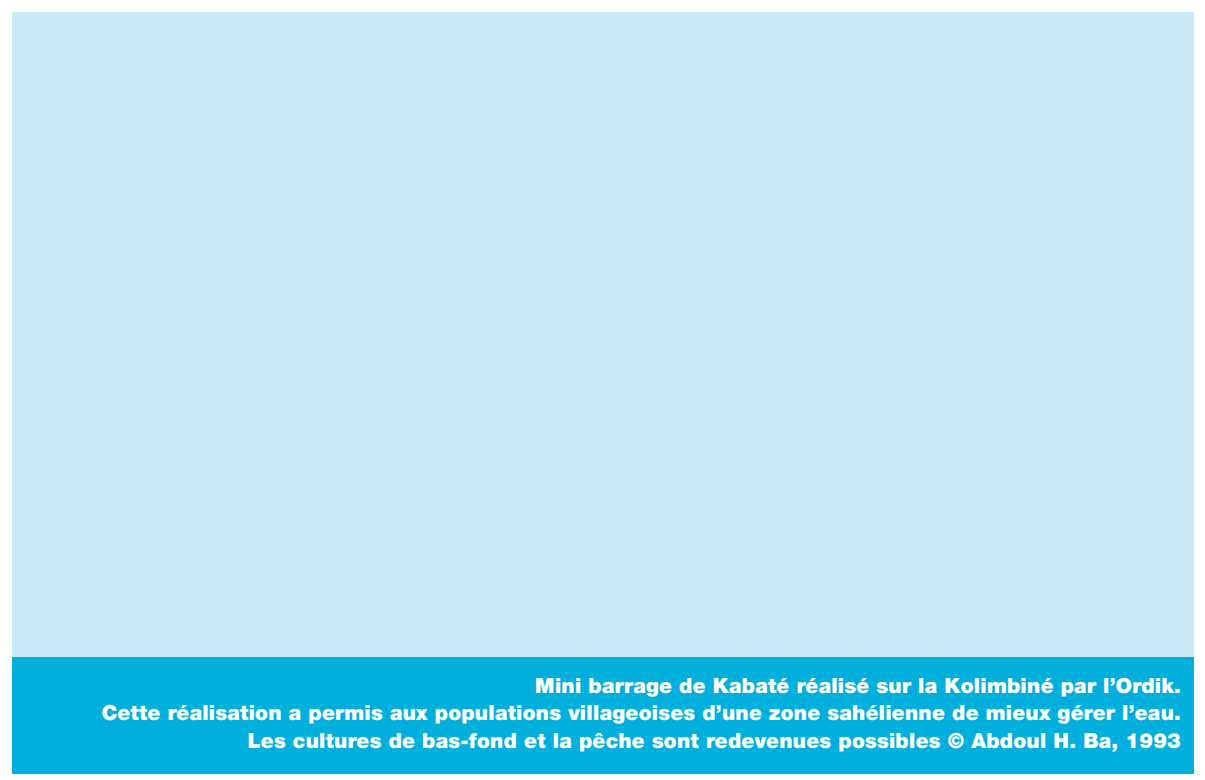


par d'anciens migrants et témoignent d'une mobilisation de compétences, d'une aptitude à transférer des savoir-faire, à activer un réseau de relations noué pendant l'immigration pour mieux exploiter et gérer une ressource naturelle locale.

Les interactions sociales et spatiales liées à ces types de projets sociaux et environnementaux sont multiples : ils permettent la préservation des écosystèmes, le maintien des savoir-faire locaux (techniques de pêche, de culture, systèmes d'élevage), les échanges des savoir-faire, la valorisation des produits locaux, la création de nouveaux emplois en milieu rural (pompiste, mécanicien, alphabétiseur en langue locale...), l'amélioration des conditions sanitaires, l'augmentation du pouvoir d'achat des villageois, le maintien de quelques jeunes dans les villages malgré la pression qu'exerce "l'imaginaire migratoire ${ }^{(3)}$ ".

\section{Coopération décentralisée et codéveloppement}

C'est vers le milieu des années quatre-vingt que la notion de coopération décentralisée s'est imposée comme une des formes des relations entre collectivités territoriales. Cette démarche s'est renforcée au début des années quatre-vingt-dix, grâce au processus d'alternance politique, de décentralisation et de régionalisation dans certains pays africains comme le Mali, la Mauritanie et le Sénégal. Mais la date clé reste l'année 1992. En effet, en France, la loi du 6 février 1992 donne un cadre légal et réglementaire à la coopération décentralisée ${ }^{(4)}$. Elle définit la relation entre des collectivités françaises et des collectivités territoriales étrangères. Le terme de "coopération" fait référence à des initiatives menées en collaboration, celui de "décentralisée" définit le niveau et le mode de cette coopération.

La coopération internationale n'est plus l'apanage de l'État. D'ailleurs nous observons un nombre croissant de collectivités territoriales françaises engagées dans des programmes de solidarité internationale. La coopération décentralisée a permis aux élus des collectivités territoriales du Nord et du Sud de développer des échanges de "proximité" pour faire face à des problèmes comme la santé et l'éducation des enfants, la gestion urbaine, l'assainissement, la démocratie locale, le développement économique local, la formation des agents municipaux, la préservation des ressources naturelles.

Dans beaucoup de cas, les programmes de solidarité ont permis d'améliorer les conditions de vie des populations du Sud (éducation sanitaire et accès à l'eau potable par exemple) et de renforcer la citoyenneté et la démocratie participative dans les villes africaines ; ils ont aussi contribué à sensibiliser les gens du Nord à la diversité 
culturelle et aux problèmes de pauvreté. Dans la plupart des cas, les collectivités locales ou territoriales sollicitent l'expertise des organisations non gouvernementales $(\mathrm{ONG})$ intervenant déjà dans les régions partenaires. Les migrants et leurs associations commencent à faire entendre leur voix dans le cadre de la coopération décentralisée mais ils restent encore marginalisés. Pourtant ils constituent un atout pour la coopération au développement.

L'État sénégalais l'a bien compris ; différents ministères travaillent ensemble pour mieux mobiliser les apports de la "diaspora". La création en 2009 du PAISD (Programme d'appui aux initiatives de solidarité pour le développement) et d'un fonds d'appui aux projets des émigrés montrent que le Sénégal entend soutenir d'une manière plus structurée les initiatives des migrants. Cependant, ces aides vont pour l'instant en majorité aux élites de cette "diaspora" dont les projets sont orientés vers le secteur tertiaire et sont réalisés dans les grandes villes comme Dakar, Thiès ou Saint-Louis.

Dans le même temps, dans les espaces ruraux, les anciennes associations de migrants sahéliens continuent à réaliser des projets non seulement à l'échelle du village d'origine, mais aussi à celle des communautés rurales. C'est le cas de l'association Urof (Union des ressortissants de Orkadiéré en France). Cette association a réalisé son premier projet en 1973 (une mosquée d'un montant de 600000 francs français), d'autres projets ont suivi (école, poste de santé, achat de pirogues...). À l'heure actuelle, l'Urof participe à hauteur de 30000 euros (soit $20 \%$ du budget total) à la réalisation d'un collège d'enseignement secondaire dans la région de Matam (vallée du fleuve Sénégal) pour la communauté rurale du même nom. Les collectivités territoriales françaises qui interviennent dans la vallée du fleuve Sénégal, comme la région NordPas-de-Calais, ne participent pas pour l'instant au financement de ce projet.

\section{L'oubli des migrants dans l'articulation du local et du global}

Cet exemple montre que les relations entre les migrants et les collectivités territoriales intervenant dans la région d'origine de ces mêmes migrants ne sont pas systématiques. On peut d'ailleurs observer une ignorance mutuelle. Les raisons de ce manque de collaboration sont multiples et varient en fonction des collectivités. En effet, les espaces de rencontre entre migrants et élus des conseils régionaux sont quasi inexistants. Par ailleurs, l'image négative qui pèse sur l'immigré ne facilite guère cet échange formel. Le migrant est encore trop perçu en Europe comme une personne à faible compétence, pauvre, venant d'un pays "sous développé". 
Cette perception négative de l'immigré ne favorise pas la reconnaissance de ses compétences dans le domaine de la conception et du financement des projets de développement local. Par ailleurs, les migrants originaires des villages ont longtemps souffert d'un manque de reconnaissance explicite de la part des élites et des élus des pays d'origine ${ }^{(5)}$. De fait les élus des collectivités et les organisations non gouvernementales ne semblent guère prêts à laisser aux migrants la place qui leur revient dans le choix et le portage des projets de solidarité. S'agit-il d'un manque de confiance ou d'une volonté des élites du Sud et des acteurs du Nord de garder le pouvoir ? La très faible prise en compte des apports des migrants par les experts et les élus du Nord dans la coopération décentralisée témoigne-t-elle de la survivance du complexe de supériorité hérité de la période coloniale ou tout simple-

Les migrants et les villages
ont su très vite privilégier
les projets les plus
consensuels possibles.
De ce point de vue,
on peut considérer qu’ils
ont développé une forme
de gouvernance
intéressante dès le milieu
des années soixante.
ment d'une vision très différente entre acteurs dans le choix des outils et des méthodes pour réaliser les projets de coopération? Pourtant les études montrent que les migrants pratiquent une double appartenance territoriale entre pays d'origine et pays d'accueil ${ }^{(6)}$. Les migrants sahéliens jouissent d'une longue expérience de mise en relation et ont une connaissance réelle des territoires et des besoins locaux des populations du Sud. En considérant les associations de migrants comme des acteurs à part entière de la coopération, les responsables des collectivités territoriales du Nord et du Sud feraient évoluer les outils méthodologiques de la coopération au développement avec l'instauration d'une gouvernance participative des migrants tant dans les pays d'origine que dans les pays d'accueil. Dans le cas du Sénégal, les migrants qui sont rentrés s'impliquent dans la vie politique et gagnent des mandats électifs (conseil rural, conseil régional, Parlement) ils continuent à jouer le rôle d'intermédiaire entre les villageois et les immigrés, entre les villageois et les élus des collectivités françaises.

\section{La force du migrant : le croisement des savoir-faire culturels}

Les trajectoires migratoires sont multiples et les investissements dans les pays d'origine peuvent prendre des formes variées. Dans tous les cas, les migrants participent à la transformation de leur espace d'origine. Ils tentent de construire ou 
de (re)construire des territoires avec des outils nouveaux fabriqués à partir du savoirfaire local préservé par ceux qui sont restés et du savoir-faire et des techniques acquis dans l'immigration ou proposés par les ONG et les collectivités territoriales occidentales. Ce processus n'est pas exempt de conflits, comme la remise en cause du pouvoir traditionnel foncier, la stratification sociale basée sur le lignage ou la perte d'influence de certains bourgs au profit des villages d'émigrés, devenus de nouveaux centres ruraux car mieux équipés ${ }^{(7)}$. Mais la volonté d'améliorer les conditions de vie prévaut et des compromis sont souvent trouvés.

Les migrants et les villages ont su très vite privilégier les projets les plus consensuels possibles. De ce point de vue, on peut considérer qu'ils ont développé une forme de gouvernance intéressante dès le milieu des années soixante. L'interaction sociale et territoriale trouve ici sa pleine expression. L'Alaef, regroupant villages maliens et sénégalais, témoigne bien de l'existence d'une coopération au développement local et d'une forme réussie de gouvernance territoriale locale dans les régions d'émigration. Les techniques de concertation, de consultation, de dialogue et de prise de décision élaborées par les migrants et les villageois sont intéressantes à plus d'un titre. C'est une rencontre entre la tradition et la modernité, c'est aussi une rencontre entre acteurs issus du même "terreau", mais avec des perceptions territoriales et des perspectives de développement parfois différentes et complémentaires. C'est cette articulation réelle des perceptions et des motivations des acteurs aux parcours variés qui singularise les zones d'émigration du Sénégal. Il n'est pas étonnant que les territoires qui se dessinent dans ce contexte augurent de nouvelles territorialités, de nouveaux rapports de l'homme à l'espace.

\section{Conclusion}

Les luttes contre la pauvreté et contre toutes les formes de discrimination sociale ou d'inégalités territoriales ainsi que le combat pour instaurer une démocratie participative et une solidarité entre les peuples sont des actions qui nécessitent la mobilisation d'acteurs divers (État, collectivités, associations...). Les migrants, par leur attachement à leur pays d'origine ont initié le codéveloppement. L'avènement des formes nouvelles de solidarité comme la coopération décentralisée permet d'agir localement au plus près des populations, d'encourager la participation de tous les acteurs, de sensibiliser à la diversité culturelle et de tendre vers des échanges réciproquement avantageux entre les peuples. Cette coopération au développement participe à la fabrication de territoires nouveaux avec des modes de gouvernance inédits. 
Mais le décalage reste grand entre l'ambition du codéveloppement et les réalités du terrain. D'abord les budgets des collectivités territoriales alloués aux actions de solidarité sont très limités alors que les partenaires du Sud sont démunis. Ensuite, il n'est pas facile d'avoir une convergence de vue sur les stratégies de développement entre les acteurs du Nord et ceux du Sud. Enfin, les migrants, malgré leur solide expérience dans le domaine du développement local et de la solidarité, restent marginalisés. Une rupture dans l'accès aux droits (droit de vote des immigrés aux élections locales en France), un accès plus simple des associations de migrants aux financements, une implication des jeunes nés en France de parents immigrés permettraient de maintenir et poursuivre les actions de développement. Dans tous les cas, par leurs actions, les migrants sahéliens montrent une forme de solidarité que la distance métrique ne semble pas altérer. Les projets des migrants sahéliens participent à la construction de territoires qui renvoient à une configuration spatiale fondée sur une sorte d'archipel dont chaque élément est relié par des réseaux territorialisés et/ou non territorialisés ${ }^{(8)}$. Les actions des migrants ici et là-bas révèlent diverses formes d'interactions spatiales et sociales, signe que les peuples et les territoires changent par les migrations.

\section{Notes}

1. Les analyses proposées dans cet article s'appuient sur des données qualitatives collectées depuis 1993 : entretiens, observations de terrain en Afrique sahélienne (Mali, Mauritanie et surtout au Sénégal), en France et en Belgique.

2. Voir Gonin Patrick et al., La Fonction émigrée dans les stratégies de développement, ENS Fontenay-Saint-Cloud, Centre de géographie rurale/CNRS-ATP, 1988.

Simon Gildas, Géodynamique des migrations internationales dans le monde, Paris, PUF, 1995.

3. Simon Gildas, La Planète migratoire dans la mondialisation, Paris, Armand Colin, 2008.

4. Cf. le Titre IV de cette loi.

5. Lors de sa visite à Lille en 1997, la délégation du Conseil régional de Saint-Louis n'avait manifesté aucune envie de rencontrer d'une manière formelle les associations des migrants originaires de la région de Saint-Louis

6. Bertini Barbara, Gonin Patrick, Kotlok Nathalie et Le Masson Olivier, "Codéveloppement et double présence : les initiatives migrantes et la fabrique des identités multiples", in S. Dessajan, N. Hossard et E. Ramos (dir.), Immigration et identité nationale. Une altéritée revisitée, Paris, L'Harmattan, 2009.

7. Ba Abdoul Hameth, Acteurs et territoires du Sahel : Rôle des mises en relations dans la recomposition des territoires, ENS Éditions, Lyon, 2007.

8. Velasco-Graciet Hélène, Territoires, mobilités et sociétés. Contradictions géographiques et enjeux pour la géographie, Paris, éditions de la Maison des sciences de l'homme, 2009. 